\title{
Successful Anesthetic Management of Patient with Stiff Person Syndrome
}

\author{
Muhammad Sohaib and Ali Sarfaraz Siddiqui
}

\begin{abstract}
We herein describe the successful anesthetic management of a patient with stiff person syndrome undergoing right total hip replacement under spinal anesthesia. We also describe the problems associated with general anesthesia. The advantage of using regional anesthesia in these patients is the avoidance of muscle relaxants. The use of general anesthesia carries the risk of hypotonia in stiff person syndrome postoperatively due to enhancement of $\gamma$ aminobutyric acid (GABA) action on synaptic transmission by drugs that have a $\gamma$ GABA agonistic action.
\end{abstract}

Key Words: Spinal anesthesia, Stiff person syndrome, General anesthesia, Hypotonia.

\section{INTRODUCTION}

Stiff Person Syndrome (SPS) is a rare autoimmune based neurological disease characterised by rigidity and painful spastic episodes primarily involving the muscles of abdomen, spine and lower limb. 1 The reported incidence of the disease is $1: 1,000,000$ and patients usually presents in 4 th decade of their life. The disease is more common in females than males with $2: 1$ ratio. ${ }^{2}$ The diagnosis of the disease is often delayed due to its rarity and non-specific signs and symptoms. Muscle spasms associated with the disease are very debilitating and fluctuating in severity and timing. These spasms may occur spontaneously or triggered by pain, anxiety and contact with the unusual surface or loud noises. ${ }^{2}$

The primary pathology is the presence of autoantibodies against glutamic acid decarboxylase 65 isoenzyme (GAD 65) and positive serology is not required for the diagnosis. ${ }^{3}$ Glutamic acid decarboxylase (GAD) is responsible for the last rate limiting step in the synthesis of gamma-aminobutyric acid (GABA). Due to loss of inhibition by GABA, there is chronic activation of motor neurons causing muscle contractions leading to muscle spasm and rigidity. SPS is also associated with other autoimmune disease like thyroiditis, diabetes mellitus, etc. 4

Presently, there is no cure for the disease but the main management relies on enhancing the GABA receptors by targeting the $\mathrm{GABA}_{B}$ through baclofen therapy. Multimodal analgesia can be used to relieve the pain. Corticosteroids, intravenous immunoglobulins and

Department of Anaesthesiology, The Aga Khan University

Hospital, Karachi, Pakistan

Correspondence: Dr. Muhammad Sohaib, Department of

Anaesthesiology, The Aga Khan University Hospital,

Stadium Road, Karachi, Pakistan

E-mail:m.sohaib@yahoo.com

Received: April 19, 2018; Accepted: July 24, 2018 plasmapheresis can be helpful in modulating the disease process. ${ }^{5}$ The anesthetic implications of SPS have been infrequently reported. Due to some anesthetic medications interacting with GABA receptors, anesthesia in SPS patients requires special attention. We describe the management of SPS patient for total hip replacement under spinal anesthesia.

\section{CASE REPORT}

A 53-year male with height of $158 \mathrm{~cm}$ and weight of $65 \mathrm{~kg}$, known case of SPS, was admitted to hospital with the complaint of shortening of right leg for 4 months and was planned for total hip replacement by orthopedic team. Actually, he was admitted in the hospital with the diagnosis of community acquired pneumonia 6 months ago. During that stay, he sustained the right femoral intertrochanteric fracture, which he noticed after the discharge from hospital as shortening of right limb at home. Patient then waited for some time as he was recovering from pneumonia and then consulted the orthopedic surgeon. Past surgical history was not significant.

He was diagnosed as a case of SPS by the neurology team about 5 years ago from the current admission. He had significant lower limb muscle spasm with history of multiple falls. He had to take help from family members to carry out routine activities and was unable to perform fine motor skills. He developed muscle contractures over the period of 2 to 3 years, which greatly impaired his mobility. He was functional class III. Patient was treated with Intravenous immunoglobulins but no remarkable improvement was noted. Currently, in this admission, he was on oral medications baclofen, tizanidine, clonazepam and gabapentin.

Physical examination of the patient revealed spasticity of lower limb along with the involvement of spinal muscles. Motor strength was fine in all four limbs along with reflexes. Electromyography (EMG) and electroencephalo- 
graphy were done and were normal. GAD6 autoantibodies levels were high. Mallampati grade of airway examination was II with normal thyromental distance. Laboratory data was within normal limits. Echocardiography (ECG) was done which showed normal ejection fraction with grade I left ventricular dysfunction.

A very detailed discussion was carried out by the anesthesia, orthopedics and neurology team. After thorough evaluation and workup, details were discussed with the patient and spinal anesthesia was planned for the procedure. General anesthesia was avoided as patient was already on muscle relaxants, so general anesthesia could have potentiated their effects in the form of delayed recovery. Standard monitoring was applied before performing the spinal anesthesia. Midazolam and ketamine was used to sedate the patient, so the left lateral position can be made for the procedure. The most difficult part was to position the patient for spinal anesthesia. Due to the involvement of the back muscles, it was very hard to make the right lateral position of the patient. After standard precautions, $25 G$ cutting needle was introduced at the level of $L 3$ and L4 after the local infiltration of the skin with $2 \%$ lidocaine. After 3 attempts, free flow of cerebrospinal fluid (CSF) was achieved and $10 \mathrm{mg}$ hyperbaric bupivacaine and $25 \mu \mathrm{g}$ fentanyl was injected in the CSF. It took about 45 minutes to position the patient and perform the spinal anesthesia. He remained hemodynamically stable after the spinal anesthesia. We also started the propofol infusion for the sedation of the patient. Midway during the procedure, orthopedic team had a bit difficulty in reducing the femur head. The smallest possible size of the femur head was tried but unsuccessful. To facilitate the surgeons, we switched off the propofol sedation and started inhalational agent sevoflurane through face mask. Surgery team was then able to reduce the femur head. After reduction of femur head, we again started propofol infusion. Patient remained vitally stable throughout the procedure. Intraoperative blood loss was around $550 \mathrm{ml}$ and urine output remained around $0.5 \mathrm{ml} / \mathrm{kg} / \mathrm{hour}$. After the procedure, patient was shifted to recovery room. Postoperative pain was managed by multimodal analgesia involving patient controlled intravenous analgesia (PCIA) tramadol, paracetamol and ketorolac. Patient was mobilised the very next day and was discharged on 5th postoperative day.

\section{DISCUSSION}

Due to extremely low incidence of the disease, limited data is available on the anesthetic management of patient with SPS.6 We used spinal anesthesia (bupivacaine and fentanyl) for total hip replacement because general anesthesia might have been associated with delayed emergence and recovery. This technique provides suitable condition for the surgery. Epidural anesthesia has been used in SPS patient for urgent cesarean-section successfully. 2 In patients with SPS, there is conflicting evidence on the use of muscle relaxants. In a case report by Obara et al., the use of vecoronium in a diagnosed case of SPS for thymectomy, six week later for emergency appendectomy and one year later for endoscopic nasal sinus surgery had no adverse effect and $25 \%$ twitch recovery time was within normal limit. 7 However, contradictory to this, in a case report by Johnson and Miller, patient was mechanically ventilated overnight due to significant residual muscle weakness in spite of the presence of all four twitches of train of four (TOF) at the end of surgery and reversal of neuromuscular blockade of vecoronium with neostigmine and glycopyrrolate. 8 In a similar report by Ferrandis et al. of a patient of SPS undergoing cardiac surgery receiving pancuronium, the patient had normal return of TOF after the first dose of pancuronium but delayed recovery. 9 In a study by Bouw et al., there was normal return of TOF but patient was ventilated for long time due to residual muscle weakness. 10 The muscle relaxants might not be the cause of residual muscle weakness after the general anesthesia. It might be the interaction between the volatile anesthetics and GABA receptors in the central nervous system leading to muscle weakness in these group of patients. The lack of objective evidence of muscle weakness in these patients is the primary reason to be cautious while administrating any type of anesthesia. The proposed hypothesis of prolonged weakness after general anesthesia is the interaction of volatile anesthetics and baclofen on GABA receptors have been documented in literature. ${ }^{11}$

Neuraxial stiffness and lumbar lordosis due to muscle spasm makes central neuraxial block difficult to perform in SPS patients. We used moderate sedation to perform spinal anesthesia as pain can exacerbate the muscle spasm in such patients. It is still debatable which anesthetic technique is safe for SPS patients. As for most rare diseases, multidisciplinary team and patient involvement can lead to the best possible outcome.

\section{REFERENCES}

1. Murinson BB. Stiff-person syndrome. Neurologist 2004; 10 : 131-7.

2. Boettcher BT, Muravyea M, Kuo C, Drexler C, Pagel PS. Anesthetic management of a parturient with stiff person syndrome for urgent cesarean delivery. Int $\mathrm{J}$ Obstet Anesth 2016; 27:85-8.

3. Neubert L, Green P, Stuart MG. Anesthetic care of stiff person syndrome in the outpatient setting. J Anesthesiol Clin Sci 2013; 2:5.

4. Baizabal-Carvallo JF, Jankovic J. Stiff-person syndrome: insights into a complex autoimmune disorder. I Neurol Neurosurg Psychiatr 2015; 86:840-8.

5. Sidransky MA, Tran NV, Kaye AD. Anesthesia considerations in stiff person syndrome. Middle East $J$ Anaesthesiol 2013; 22:217-21. 
6. Yagan O, Özyilmaz K, Özmaden A, Sayin Ö, Hanci V. Anesthesia in a patient with stiff person syndrome. Braz $J$ Anesthesiol 2016; 66:543-5.

7. Obara M, Sawamura S, Chinzei M, Komatsu K, Hanaoka K. Anaesthetic management of a patient with stiff person syndrome. Anaesthesia 2002; 57:501-21.

8. Johnson JO, Miller KA. Anesthetic implications in stiff-person syndrome. Anesth Analg 1995; 80:612-3.
9. Ferrandis R, Belda J, Llau JV, Belda C, Bahamonde JA. Anesthesia for cardiac surgery on a patient with stiff person syndrome. J Cardiothorac Vasc Anesth 2005; 19:370-2.

10. Bouw J, Leendertse K, Tijssen MA, Dzoljic M. Stiff person syndrome and anesthesia: case report. Anesth Analg 2003; 97:486-7.

11. Hylan K, Vu AD, Stammen K. Anesthetic considerations of stiff person syndrome: A case report. AANA J 2016; 84:181-7.

.......... 